\title{
Experimental Evolution of a Dense Cluster of Residues in Tyrosyl-tRNA Synthetase: Quantitative Effects on Activity, Stability and Dimerization
}

\section{Young Chul Park, Valérie Guez and Hugues Bedouelle*}

Groupe d'Ingénierie des

Protéines (CNRS URA 1129)

Unité de Biochimie Cellulaire

Institut Pasteur, 28 rue du

Docteur Roux, 75724, Paris

Cedex 15, France

\begin{abstract}
A dense cluster of eight residues was identified at the crossing of two $\alpha$-helices in tyrosyl-tRNA synthetase (TyrRS) from the thermophile Bacillus stearothermophilus. Its mechanism of evolution was characterized. Four residues of this cluster are not conserved in TyrRS from the mesophile Escherichia coli. The corresponding mutations were constructed in $\operatorname{TyrRS}(\Delta 1)$, a derivative of TyrRS from $B$. stearothermophilus in which the anticodon binding domain is deleted. Mutations I52L (i.e. Ile52 into Leu), M55L and L105V did not affect the activity of $\operatorname{TyrRS}(\Delta 1)$ in the pyrophosphate exchange reaction whereas T51P increased it. The kinetic stabilities of $\operatorname{TyrRS}(\Delta 1)$ and its mutant derivatives at $68.5^{\circ} \mathrm{C}$ were determined from experiments of irreversible thermal precipitation. They were in the order $\mathrm{L} 105 \mathrm{~V}<\mathrm{I} 52 \mathrm{~L}<\mathrm{T} 51 \mathrm{P}<$ Wild Type $\leqslant \mathrm{M} 55 \mathrm{~L}$; mutation $\mathrm{I} 52 \mathrm{~L}$ partially compensated L105V in these experiments whereas M55L was coupled neither to I52L nor to L105V. Mutations I52L and L105V affected the stability of the dimeric $\operatorname{TyrRS}(\Delta 1)$ at different steps of its unfolding by urea, monitored under equilibrium conditions by spectrofluorometry or size exclusion chromatography. I52L destabilized the association between the subunits even though residue Ile52 is more than $20 \AA$ away from the subunit interface. L105V destabilized the monomeric intermediate of unfolding. The two mutational pathways, going from the wildtype TyrRS $(\Delta 1)$ to the I52L-L105V double mutant through each of the single mutants were not equivalent for the stability of the monomeric intermediate and for the total stability of the dimer. One pathway contained two neutral steps whereas the other pathway contained a destabilizing step followed by a stabilizing step. Mutation I52L allowed L105V along the first pathway and compensated it along the second pathway. Thus, the effects of I52L and L105V on stability depended on the structural context. The gain in activity due to T51P was at the expense of a slight destabilization.
\end{abstract}

(C) 1999 Academic Press

Keywords: aminoacyl-tRNA synthetase; context effect; dimeric protein; molecular evolution; protein stability and folding

\section{Introduction}

The use of mutations to modify local interactions in proteins or as local probes of their three-dimen-

Present address: Y. C. Park, Cornell University Medical College, E-023, 1300 York Avenue, New York, NY 10021, USA.

Abbreviations used: Bst-TyRS, tyrosyl-tRNA synthetase from B. stearothermophilus; Eco-TyrRS, TyrRS from E. coli; SEC, size-exclusion chromatography.

E-mail address of the corresponding author: hbedouel@pasteur.fr sional structure, has led to great progress in the understanding of protein stability and folding. The quantitative analysis of the effects of such mutations on the unfolding equilibria has enabled one to characterize the nature and energy of the forces that stabilize the folded state of proteins (Shortle, 1989; Alber, 1989; Matthews, 1993; Fersht \& Serrano, 1993; Sturtevant, 1994; Baldwin \& Matthews, 1994). The quantitative analysis of their effects on the energy levels of the different conformational states during folding, by a combination of kinetics and equilibrium experiments, has enabled one to compare the conformations of these 
states and to deduce folding mechanisms. These analyses have been performed mainly on small monomeric proteins, they have given access to a structural description of the transition state for folding, and they have shown that folding occurs through a mechanism of simultaneous nucleation and condensation (Fersht, 1997). The existence and the role of early intermediates in the folding of more complex monomeric proteins is well documented (Roder \& Colon, 1997).

In principle, these types of mutational analyses could be extended to dimeric proteins. A prerequisite consists in being able to analyse their unfolding equilibria quantitatively and measure the effects of mutations on these equilibria with precision. This prerequisite is well established for dimeric proteins that unfold without intermediate between the native and unfolded states under equilibrium conditions (Neet \& Timm, 1994). In contrast, only a handful of papers have reported quantitative analyses of the unfolding equilibria for dimeric proteins that unfold through a dimeric or a monomeric intermediate (Clark et al., 1993; Cheng et al., 1993; Eftink et al., 1994; Grimsley et al., 1997; Park \& Bedouelle, 1998). To our knowledge, no detailed study has yet been published on the variations of stability induced by mutations in these types of proteins.

In a recent study, we have developed a coherent set of equations to analyse the unfolding equilibria of dimeric proteins quantitatively. We have applied them to tyrosyl-tRNA synthetase from Bacillus stearothermophilus (Bst-TyrRS) and established that its unfolding by urea involves a dissociation of the dimer followed by the unfolding of the monomer (Park \& Bedouelle, 1998). The quality of the data that we obtained for the wild-type BstTyrRS, suggested to us that it should be possible to quantify the effects of mutations on its stability. We were interested in a tertiary interaction at the crossing of two $\alpha$-helices, between residues Ile52 and Leu105 of Bst-TyrRS (Figure 1). These two residues are not conserved when going from $B$. stearothermophilus, which is a thermophile, to Escherichia coli, which is a mesophile. A previous study had suggested that the two corresponding changes could have compensatory effects either on the activity or on the stability of tyrosyl-tRNA synthetase (TyrRS), but the demonstration was lacking (Guez-Ivanier et al., 1993).
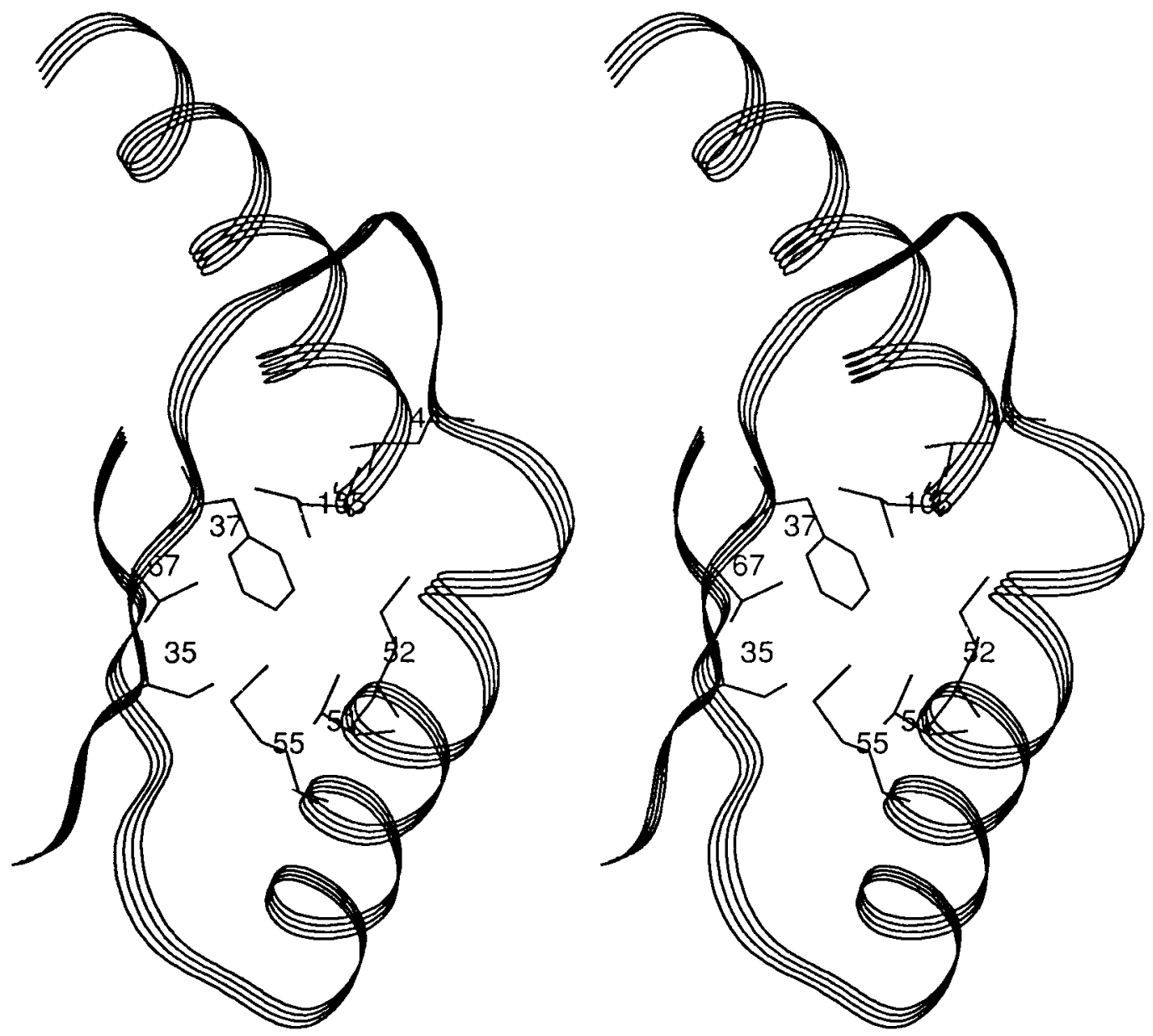

Figure 1. Dense cluster of side-chains under study in the crystal structure of Bst-TyrRS( $\Delta 1)$ (stereo view). The elements of secondary structure that contain the residues of the cluster and the side-chains of these residues are represented. The Figure was drawn with the coordinates of subunit A in the PDB entry 4 ts1. 
An analysis of the structural environment of residues Ile52 and Leu105 showed that they belong to a dense cluster of eight residues. Four residues of this cluster are not conserved between Bst-TyrRS and the tyrosyl-tRNA synthetase from E. coli (Eco-TyrRS): Thr51, Ile52, Met55 and Leu105 in Bst-TyrRS are replaced by Pro53, Ile55, Leu58 and Leu108, respectively, in Eco-TyrRS. It has been proposed that the dense clusters of residues play a role in the stability of proteins (Heringa \& Argos, 1991). However, this role has not been tested experimentally, with one exception (Heringa et al., 1995). The existence of clusters of residues involved in tertiary interactions, poses the problem of their mode of evolution since it is generally assumed that evolution occurs through sequential single mutations (see Ninio, 1996). One of the means to solve this paradox is to reconstitute the different mutational pathways that lead from a protein to a homologous protein and to compare the properties of the mutational intermediates in these pathways (Malcolm et al., 1990; Hardies \& Garvin, 1991).

Here, we studied the dense cluster of residues that includes Ile52 and Leu105 in Bst-TyrRS. We tested if the changes of residues and the resulting changes of interactions between residues in this cluster play a part in the difference of stability between Bst-TyrRS and Eco-TyrRS. We also tried to characterize the evolutionary pathway that this cluster has followed to go from its composition in B. stearothermophilus to that in E. coli. To answer these questions, we introduced mutations T51P (Thr51 into Pro), I52L, M55L and L105V in BstTyrRS, individually or in combinations. We measured and compared the effects of these mutations on the activity of Bst-TyrRS in experiments of enzyme kinetics, on its kinetic stability in experiments of irreversible thermal precipitation, and on its thermodynamic stability in experiments of unfolding by urea, monitored by spectrofluorometry or size exclusion chromatography under equilibrium conditions. We made the following observations. Mutation M55L was practically neutral; T51P increased the activity of Bst-TyrRS at the expense of a slight destabilization; I52L destabilized the association between the subunits of the Bst-TyrRS dimer even though residue Ile52 is far from their interface; the effects of I52L and L105V depended on their structural context, i.e. on the neighbouring side-chains and on the folding state of Bst-TyrRS. We concluded that the molecular evolution of a dense cluster may follow a preferential mutational pathway.

\section{Results}

\section{Identification of a dense cluster around residues Ile52 and Leu105}

We used the CLUSPROT program to analyse the structural environment of residues Ile52 and Leu105 in Bst-TyrRS. This program detects dense clusters of residues in proteins. By definition, each residue of a dense cluster makes contacts with at least two other residues of the same cluster, at distances shorter than $4.5 \AA$ A. Moreover, it makes more contacts with the other residues of the cluster than with the surrounding residues. Only the contacts between side-chains are usually considered (Heringa \& Argos, 1991; Materials and Methods). We used two crystal structures for this analysis: the structure of the full-length Bst-TyrRS (residues 1 to 419), whose two subunits are identical and symmetrical around a 2-fold rotational axis, and the structure of the truncated derivative BstTyrRS( $\Delta 1$ ) (residues 1-319), whose two subunits $A$ and $\mathrm{B}$ have slightly different conformations. Residues 320-419 are not resolved in the crystal structure of Bst-TyrRS and they are deleted in BstTyrRS( $\Delta 1)$ (Brick \& Blow, 1987; Brick et al., 1989). We found that Ile52 and Leu105 belonged to a dense cluster of ten residues in the subunit of BstTyrRS (made up of Cys35, Phe37, Leu44, His48, Thr51, Ile52, Met55, Ala67, Leu105 and Leu109). This cluster was reduced to eight residues in subunit A of Bst-TyrRS( $\Delta 1$ ) (by loss of His48 and Leu109) and was split into three small clusters in subunit B. We carefully compared the values that the interatomic distances and the torsion angles of the side-chains take in the three independent structures of the TyrRS subunit, to understand the cause of the above variations in the environment of residues Ile52 and Leu105. This comparison suggested that the variations could be due to the positional errors in the crystallographic coordinates that are expected at the resolution of the structures, $2.3 \AA$. In Bst-TyrRS, the ten residues of the dense cluster that includes Ile52 and Leu105, belong either to the super-secondary structure that is formed by $\beta$-strand $\mathrm{B}, \alpha$-helix $\mathrm{H} 3$ and $\beta$-strand $\mathrm{C}$, or to $\alpha$-helix H5 and the following loop (Figure 1). Several residues of this cluster are directly involved in the binding of the ribose of ATP (Cys35, His48, Thr51). Several others are covalently linked to residues that are involved in the binding of tyrosine (Cys35, Phe37, Ala67) or of ATP (Phe37, Leu44, Ile52; Fersht, 1987; Brick \& Blow, 1987; Brick et al., 1989).

Only four residues of the dense cluster that includes Ile52 and Leu105 in Bst-TyrRS, are not conserved in Eco-TyrRS: Thr51, Ile52, Met55 and Leu105. The side-chains of Ile52 and Leu105 form contacts at $4.0 \AA$, below the threshold of $4.5 \AA$. The side-chain of Met55 forms contacts with those of Ile52 and Leu105, at $4.6 \AA$ and $4.7 \AA$, respectively, just above the threshold. The side-chain of Thr51 is at large distances from Met55 and Leu105. Ile52, Met55 and Leu105 are hydrophobic and fully buried inside the structure; in contrast, Thr51 is polar and its side-chain is partially exposed to the solvent. We changed these four residues of Bst-TyrRS into their homologues in Eco-TyrRS, individually or in combinations, to test the importance of each of them for the activity and the stability of BstTyrRS, and to analyse the mechanisms through which a dense cluster of residues evolves. Because 
Table 1. Kinetic parameters for pyrophosphate exchange by $\operatorname{TyrRS}(\Delta 1)$ and its mutant derivatives

\begin{tabular}{|c|c|c|c|c|c|c|}
\hline Mutation & $K_{\mathrm{M}}(\mu \mathrm{M})$ & $\begin{array}{c}\text { Tyr } \\
k_{\text {cat }}\left(\mathrm{s}^{-1}\right)\end{array}$ & $k_{\text {cat }} / K_{\mathrm{M}}\left(\mathrm{s}^{-1} \mathrm{M}^{-1}\right)$ & $K_{\mathrm{M}}(\mathrm{mM})$ & $\begin{array}{c}\mathrm{ATP} \\
k_{\text {cat }}\left(\mathrm{s}^{-1}\right)\end{array}$ & $k_{\text {cat }} / K_{\mathrm{M}}\left(\mathrm{s}^{-1} \mathrm{M}^{-1}\right)$ \\
\hline WT & 2.1 & 5.4 & 2.5 & 2.1 & 7.7 & 3.6 \\
\hline I52L & 1.8 & 3.6 & 2.0 & 2.4 & 6.8 & 2.9 \\
\hline L105V & 2.1 & 4.9 & 2.3 & 2.7 & 12.0 & 4.5 \\
\hline M55L & 1.4 & 2.5 & 1.8 & 2.0 & 4.6 & 2.3 \\
\hline
\end{tabular}

The rate constants $k_{\text {cat }}$ are quoted per mol of dimeric enzyme, as measured by active site titration. Tyr, [ATP] $=2 \mathrm{mM}$ and [Tyr] between 0.5 and $50 \mu \mathrm{M}$. ATP, $[\mathrm{Tyr}]=50 \mu \mathrm{M}$ and [ATP] between 0.5 and $10 \mathrm{mM}$. The concentration of purified synthetase in the reaction was equal to $0.1 \mu \mathrm{M}$ and $\left[\mathrm{MgCl}_{2}\right]=[\mathrm{ATP}]+10 \mathrm{mM}$.

Thr51 appeared peripheral to the cluster, we restricted our analysis of the multiple mutations to those involving residues Ile52, Met55 and Leu105. We used the truncated enzyme Bst-TyrRS $(\Delta 1)$ in this work rather than the full-length Bst-TyrRS, because its unfolding can be analysed quantitatively and its crystal structure is fully known (Park \& Bedouelle, 1998; Brick et al., 1989). The changes of residues were constructed by site-directed mutagenesis of phage M13-BY $(\Delta 1)$. The mutant derivatives of Bst-TyrRS $(\Delta 1)$ were produced by infection of E.coli cells with the mutant phages.

\section{Activity of the mutant TyrRSs}

$\operatorname{TyrRS}(\Delta 1)$ does not bind and does not charge tRNA $^{\text {Tyr }}$, but it forms tyrosyl-adenylate normally. Its stoechiometry of tyrosyl-adenylate formation (one molecule of tyrosyl-adenylate per molecule of enzyme dimer) and its activity can be measured by active site titration and by the pyrophosphate exchange reaction, respectively (Waye et al., 1983). Mutations I52L, M55L and L105V did not affect the kinetic parameters of $\operatorname{TyrRS}(\Delta 1)$ in the pyrophosphate exchange reaction, i.e. $K_{M}$ for tyrosine, $K_{M}$ for ATP and $k_{\text {cat }} / K_{\mathrm{M}}$ (Table 1). Therefore, the mutations did not modify the topology of the active site and the overall conformation of TyrRS $(\Delta 1)$. The effects of mutation $\mathrm{T} 51 \mathrm{P}$ on the kinetic parameters and on the crystal structure of $\operatorname{TyrRS}(\Delta 1)$ have been reported previously. T51P decreases 130 -fold $K_{\mathrm{M}}$ for ATP, 16-fold $K_{\mathrm{M}}$ for tyrosine and increases between 25 and 50 -fold $k_{\text {cat }} / K_{\mathrm{M}}$ (Wilkinson et al., 1984). It does not induce any structural rearrangement, except for the local change of the side-chain (Brown et al., 1987). Thus, three of the changes had no significant effect on the activity of $\operatorname{TyrRS}(\Delta 1)$ and the fourth one improved it. These results excluded the possibility that these four residues had co-evolved to preserve the activity of TyrRS, at least at the level of tyrosine activation. We therefore tested the effect of their changes, either singly or in combinations, on stability (see below). Active site titration of the multiple mutants showed that they were functional for the formation of tyrosyl-adenylate and therefore correctly folded.

\section{Unfolding by thermal treatment}

We compared the kinetics of irreversible thermal precipitation for the wild-type $\operatorname{TyrRS}(\Delta 1)$ and for its mutant derivatives to test whether the mutations altered its stability. The fluorescence intensity of the soluble fraction decayed exponentially with time in these experiments, whatever the TyrRS( $\Delta 1$ ) derivative (Figure 2). Table 2 gives the values of the corresponding rate constant, for kinetics performed at $68.5^{\circ} \mathrm{C}$ and with an initial protein concentration of $100 \mu \mathrm{g} / \mathrm{ml}(1.4 \mu \mathrm{M})$. We assumed that the soluble molecules of $\operatorname{TyrRS}(\Delta 1)$ were in a dimeric state and that the fluorescence intensity was proportional to the concentration of dimer in the conditions of the measurement, $25^{\circ} \mathrm{C}$ in water. We then applied the transition state theory to translate the rate constant of precipitation into a free energy of activation, $\Delta G^{*}$, which is a measure of the kinetic stability (Pappenberger et al., 1997). From the values of $\Delta G^{\sharp}$, we calculated the variations $\Delta \Delta G^{*}$ of free energy when going from the wild-type $\operatorname{TyrRS}(\Delta 1)$ to each of its mutants (Table 2). The single mutations ranked in the following order according to the values of $\Delta \Delta G^{*}$, i.e. to their destabilizing effects: M55L $\leqslant$ WT $<$ T51P $<$ I52L $<$ L105V.

For each couple of mutations, we also calculated the energetic effect $\Delta \Delta G_{\mathrm{A}}^{\star}$ of the less damaging

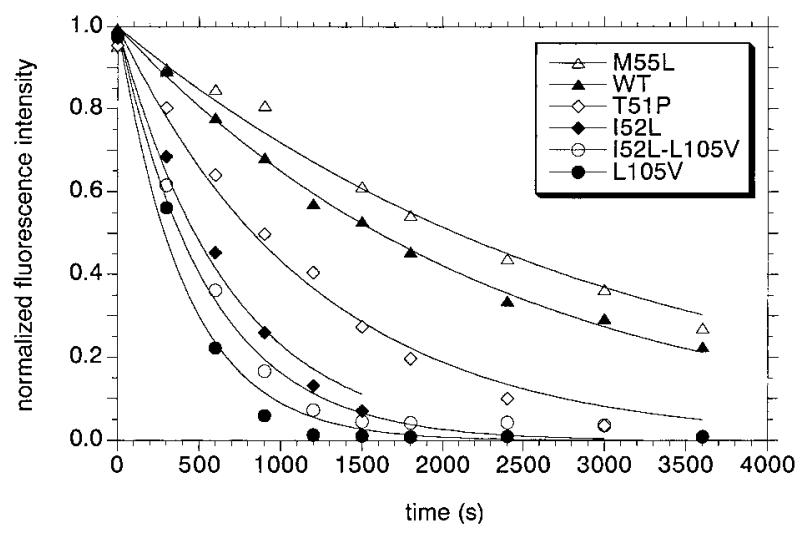

Figure 2. Kinetics of thermal precipitation for $\operatorname{TyrRS}(\Delta 1)$ and its mutant derivatives at $68.5^{\circ} \mathrm{C}$. The initial concentration of purified synthetase was $100 \mu \mathrm{g} /$ $\mathrm{ml}(1.4 \mu \mathrm{M})$. The kinetics were followed by the fluorescence intensity $Y$ of the soluble protein fraction. The curves were obtained by fitting equation (1) to the experimental data. The fluorescence intensities are normalized. See Materials and Methods for details. Only part of the time points were represented and the curves for the multiple mutations I52L-M55L, M55L-L105V and I52L-M55L-L105V were omitted for clarity. 
Table 2. Rate constants and free energies of activation for the thermal precipitation of TyrRS $(\Delta 1)$ and its mutant derivatives at $68.5^{\circ} \mathrm{C}$

\begin{tabular}{|c|c|c|c|c|}
\hline Mutation & $k \pm \operatorname{SE}\left(10^{-4} \mathrm{~s}^{-1}\right)$ & $R_{\mathrm{P}} \pm \mathrm{SE} * 10^{3}$ & $\Delta G^{*} \pm \mathrm{SE}\left(\mathrm{kcal} \mathrm{mol}^{-1}\right)$ & $\Delta \Delta G^{*} \pm \mathrm{SE}\left(\mathrm{kcal} \mathrm{mol}^{-1}\right)$ \\
\hline WT & $4.58 \pm 0.61$ & $997 \pm 1$ & $25.334 \pm 0.081$ & $0.00 \pm 0.11$ \\
\hline T51P & $8.32 \pm 0.15$ & $993 \pm 1$ & $24.907 \pm 0.012$ & $0.43 \pm 0.08$ \\
\hline M55L & $3.30 \pm 0.28$ & $990 \pm 6$ & $25.545 \pm 0.059$ & $-0.21 \pm 0.10$ \\
\hline L105V & $23.4 \pm 0.2$ & $983 \pm 1$ & $24.206 \pm 0.006$ & $1.13 \pm 0.08$ \\
\hline I52L-M55L & $9.01 \pm 0.07$ & $994 \pm 1$ & $24.854 \pm 0.005$ & $0.48 \pm 0.08$ \\
\hline I52L-L105V & $17.9 \pm 0.2$ & $993 \pm 2$ & $24.389 \pm 0.008$ & $0.94 \pm 0.08$ \\
\hline M55L-L105V & $14.7 \pm 0.7$ & $994 \pm 1$ & $24.521 \pm 0.031$ & $0.81 \pm 0.09$ \\
\hline I52L-M55L-L105V & $14.7 \pm 1.1$ & $994 \pm 2$ & $24.526 \pm 0.055$ & $0.81 \pm 0.10$ \\
\hline
\end{tabular}

Each entry gives the average value and the associated standard error (SE) in at least three independent experiments. $k$, rate constant; $R_{\mathrm{P}}$, Pearson coefficient for the fitting of equation (1) to the experimental data; $\Delta G^{*}$, free energy of activation calculated by equation (2); $\Delta \Delta G^{*}$, variation of $\Delta G^{*}$ on mutation, calculated by equation (17). The SE values on $\Delta \Delta G^{*}$ were deduced from the SE values on $\Delta G^{\star}$ by equation (21). See Materials and Methods for details.

mutation in the context of the more damaging one and the energy of coupling $\Delta \Delta G_{\mathrm{B}}^{\mathrm{B}}$ between the two mutations (Table 3; Mildvan et al., 1992; Fersht et al., 1992). The values of $\Delta \Delta G_{B}^{*}$ showed that mutations M55L and I52L on the one hand, M55L and L105V on the other hand, had additive effects on stability, whereas I52L and L105V had non-additive effects. A comparison of the values of $\Delta \Delta G^{\ddagger}$ and $\Delta \Delta G_{\mathrm{A}}^{\ddagger}$ (Tables 2 and 3) showed that mutation M55L had nearly the same effects in the contexts of the wild-type $\operatorname{TyrRS}(\Delta 1)$, of the I52L and L105V single mutants, and of the I52LL105V double mutant. This comparison also showed that mutation I52L had a strongly destabilizing effect in the context of the wild-type $\operatorname{TyrRS}(\Delta 1)$ and a stabilizing effect in the context of the L105V mutant. We concluded that the contacts between residues Met55 and either Ile52 or Leu105 were not important for the kinetic stability of $\operatorname{TyrRS}(\Delta 1)$, that the interaction between Ile52 and Leu105 was important, and that mutation I52L partially compensated (suppressed) the destabilizing effect of L105V. On the basis on these results, we decided to concentrate our study on mutations I52L and L105V and to further test their effects on stability by experiments of unfolding with urea in equilibrium conditions.

\section{Unfolding by urea, monitored by fluorescence intensity}

Previously, we have studied the unfolding of the wild-type $\operatorname{TyrRS}(\Delta 1)$ by urea. We have determined conditions in which its thermodynamic equilibrium of unfolding is reached and found that its unfolding is reversible under these conditions (GuezIvanier \& Bedouelle, 1996). We have shown that $\operatorname{TyrRS}(\Delta 1)$ unfolds through a monomeric state, intermediate between the native dimeric state and the unfolded state, according to the mechanism $\mathrm{N}_{2} \Longleftrightarrow$ 2I $\Longleftrightarrow 2 \mathrm{U}$ (Park \& Bedouelle, 1998). In particular, we have monitored the unfolding of $\operatorname{TyrRS}(\Delta 1)$ by its intensity of fluorescence. From the unfolding profiles thus obtained, we have quantified the following thermodynamic parameters: $\Delta G_{1}\left(\mathrm{H}_{2} \mathrm{O}\right)$, the free energy for the dissociation of the dimer subunits; $\Delta G_{2}\left(\mathrm{H}_{2} \mathrm{O}\right)$, the free energy for the unfolding of the monomeric intermediate; $\Delta G\left(\mathrm{H}_{2} \mathrm{O}\right)$, the total free energy of unfolding; and $m_{1}, m_{2}$ and $m$, the coefficients of dependence of these free energies on the concentration of urea (Park \& Bedouelle, 1998). Here, we monitored the unfolding of the $\operatorname{TyrRS}(\Delta 1)$ derivatives that carried the single mutations T51P, I52L or L105V, or the double mutation I52L-L105V, by their intensities of fluorescence in identical exper-

Table 3. Coupling parameters between mutations I52L, M55L and L105V in the experiments of thermal precipitation

\begin{tabular}{lccr}
\hline mut $_{1}$ & mut $_{2}$ & $\Delta \Delta G_{\mathrm{A}}^{ \pm} \pm \mathrm{SE}\left(\mathrm{kcal} \mathrm{mol}^{-1}\right)$ & $\Delta \Delta G_{\mathrm{B}}^{*} \pm \mathrm{SE}\left(\mathrm{kcal} \mathrm{mol}^{-1}\right)$ \\
\hline I52L & M55L & $-0.29 \pm 0.07$ & $-0.08 \pm 0.12$ \\
L105V & I52L & $-0.18 \pm 0.01$ & $-0.95 \pm 0.11$ \\
L105V & M55L & $-0.32 \pm 0.03$ & $-0.10 \pm 0.11$ \\
M55L-L105V & I52L & $0.00 \pm 0.06$ & $-0.77 \pm 0.12$ \\
L105V & I52L-M55L & $-0.32 \pm 0.06$ & $-0.80 \pm 0.10$ \\
I52L-L105V & M55L & $-0.14 \pm 0.06$ & $0.07 \pm 0.11$ \\
\hline
\end{tabular}

mut $_{1}$ and mut $_{2}$, the more and the less damaging mutations in the context of the wild-type TyrRS $(\Delta 1) ; \Delta \Delta G_{\mathrm{A}}^{\star}$, effect of mut ${ }_{2}$ in the context of mut m $_{1}$, calculated by equation (19); $\Delta \Delta G_{\mathrm{B}}^{ \pm}$, free energy of coupling between the effects of mut ${ }_{1}$ and mut and $_{2}$ calculated by equation (20). The SE values on $\Delta \Delta G_{\mathrm{A}}^{\star}$ and $\Delta \Delta G_{\mathrm{B}}^{\ddagger}$ were calculated from the SE values on $\Delta G^{\ddagger}$ (Table 2) by equation (21). See Materials and Methods for details. 
WT

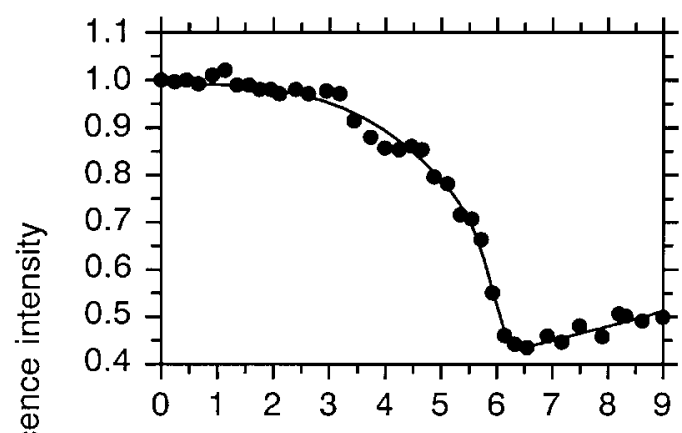

L105V

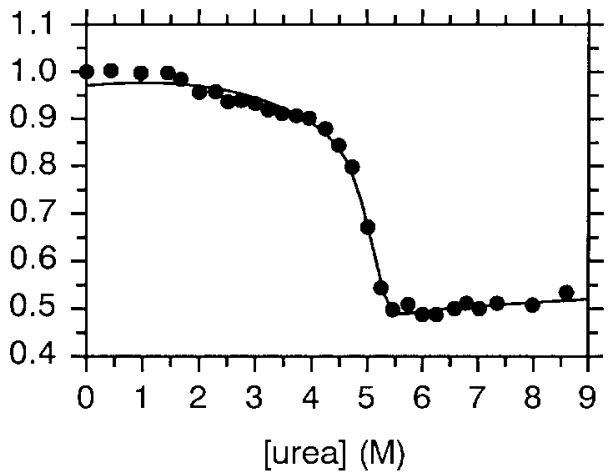

$152 \mathrm{~L}$

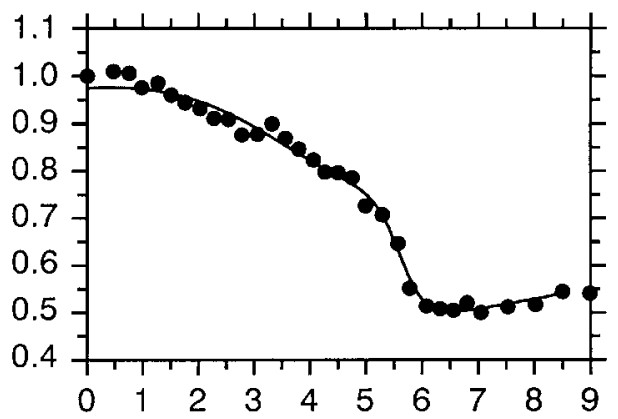

I52L-L105V

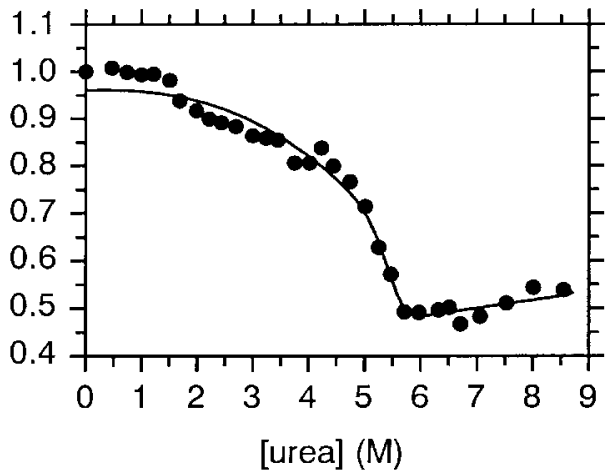

Figure 3. Unfolding of TyrRS $(\Delta 1)$ and its mutant derivatives by urea, as monitored by their fluorescence intensity (excitation at $278 \mathrm{~nm}$, emission at $330 \mathrm{~nm}$ ). The concentration of purified synthetase was $10 \mu \mathrm{g} / \mathrm{ml}(138 \mathrm{nM})$. The signal is given along the $y$ axis and expressed as a fraction of the native protein signal in the absence of urea. The continuous line was obtained by fitting equation (16) to the experimental data. See Materials and Methods for details.

iments (Figure 3). A visual inspection revealed that mutation I52L affected the unfolding profile mainly between 2 and $5 \mathrm{M}$ urea, L105V between 5 and $6 \mathrm{M}$ urea, and I52L-L105V in an intermediate way. We fitted equation (16), which links the global intensity of protein fluorescence and the concentration of urea, to each of the unfolding profiles to quantify the effects of the mutations (Materials and Methods). Table 4 gives the thermodynamic parameters resulting from these fittings, for each of the mutant derivatives of $\operatorname{TyrRS}(\Delta 1)$.
From the free energies of unfolding, we calculated their variations between the wild-type $\operatorname{TyrRS}(\Delta 1)$ and each mutant derivative. The variations of free energies due to mutation $\mathrm{T} 51 \mathrm{P}$ had the following values: $\Delta \Delta G_{1}\left(\mathrm{H}_{2} \mathrm{O}\right)=0.8( \pm 0.2) \mathrm{kcal}$ $\mathrm{mol}^{-1}, \quad \Delta \Delta G_{2}\left(\mathrm{H}_{2} \mathrm{O}\right)=0.7( \pm 1.0) \mathrm{kcal} \mathrm{mol}^{-1}$ and $\Delta \Delta G\left(\mathrm{H}_{2} \mathrm{O}\right)=2( \pm 2) \mathrm{kcal} \mathrm{mol}^{-1}$. Thus, mutation T51P significantly destabilized the association between the subunits of $\operatorname{TyrRS}(\Delta 1)$. The variations of free energies due to mutations I52L, L105V and I52L-L105V are given in Figure 4, in the form of

Table 4. Thermodynamic parameters for the unfolding of $\operatorname{TyrRS}(\Delta 1)$ and its mutant derivatives by urea, as monitored by their fluorescence intensity

\begin{tabular}{lccccc}
\hline Parameter & I52L & L105V & I52L-L105V & T51P & WT \\
\hline$m_{1}\left(\mathrm{kcal} \mathrm{mol}^{-1} \mathrm{M}^{-1}\right)$ & $0.763 \pm 0.042$ & $0.83 \pm 0.14$ & $0.657 \pm 0.055$ & $0.876 \pm 0.032$ & $0.904 \pm 0.060$ \\
$\Delta G_{1}\left(\mathrm{H}_{2} \mathrm{O}\right)\left(\mathrm{kcal} \mathrm{mol}^{-1}\right)$ & $11.315 \pm 0.053$ & $13.10 \pm 0.43$ & $11.663 \pm 0.092$ & $12.983 \pm 0.065$ & $13.75 \pm 0.16$ \\
$m_{2}\left(\mathrm{kcal} \mathrm{mol}^{-1} \mathrm{M}^{-1}\right)$ & $2.654 \pm 0.050$ & $2.13 \pm 0.18$ & $2.780 \pm 0.096$ & $2.53 \pm 0.18$ & $2.476 \pm 0.085$ \\
$\Delta \mathrm{G}_{2}\left(\mathrm{H}_{2} \mathrm{O}\right)\left(\mathrm{kcal} \mathrm{mol}^{-1}\right)$ & $15.20 \pm 0.50$ & $11.05 \pm 0.83$ & $15.48 \pm 0.69$ & $13.19 \pm 0.80$ & $13.87 \pm 0.59$ \\
$m\left(\mathrm{kcal} \mathrm{mol}^{-1} \mathrm{M}^{-1}\right)$ & $6.071 \pm 0.059$ & $5.09 \pm 0.36$ & $6.22 \pm 0.25$ & $5.93 \pm 0.33$ & $5.86 \pm 0.12$ \\
$\Delta G\left(\mathrm{H}_{2} \mathrm{O}\right)\left(\mathrm{kcal} \mathrm{mol}^{-1}\right)$ & $41.7 \pm 1.0$ & $35.2 \pm 1.6$ & $42.6 \pm 1.5$ & $39.4 \pm 1.5$ & $41.5 \pm 1.1$ \\
\hline
\end{tabular}

The unfolding of the synthetases was monitored as described in the legend to Figure 3. $\Delta G_{1}\left(\mathrm{H}_{2} \mathrm{O}\right), \Delta G_{2}\left(\mathrm{H}_{2} \mathrm{O}\right)$ and $\Delta G\left(\mathrm{H}_{2} \mathrm{O}\right)$, variations of free energy between states $\mathrm{N}_{2}$ and $2 \mathrm{I}, \mathrm{I}$ and $\mathrm{U}$, and $\mathrm{N}_{2}$ and $2 \mathrm{U}$, respectively, as defined by equations (6) to (8). $m_{1}, m_{2}$ and $m$, coefficients of dependence of the variations of free energy on the concentration of urea, as defined by the same equations. $\Delta G_{1}\left(\mathrm{H}_{2} \mathrm{O}\right), \Delta G_{2}\left(\mathrm{H}_{2} \mathrm{O}\right), m_{1}$ and $m_{2}$ were obtained by fitting equation (16) to the experimental data. $\Delta G\left(\mathrm{H}_{2} \mathrm{O}\right)$ and $m$ were calculated by equation (7). Each entry gives the average value and the associated SE in at least two independent experiments. See Materials and Methods for details. 

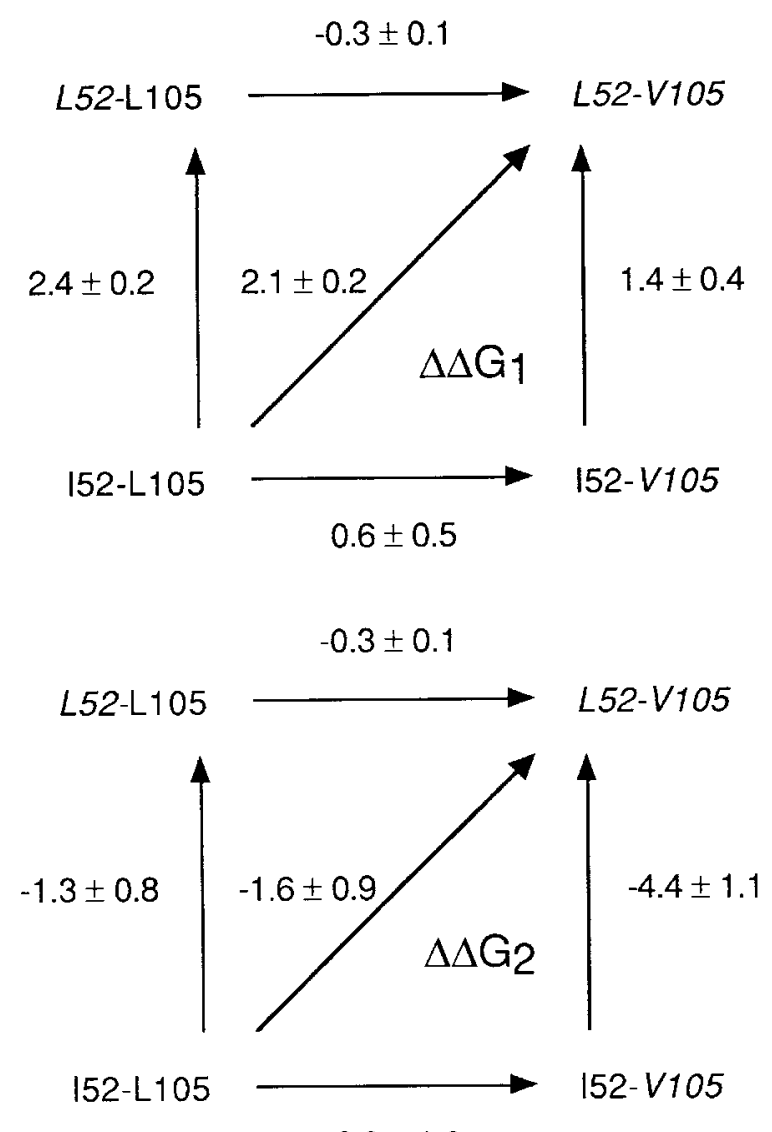

$2.8 \pm 1.0$

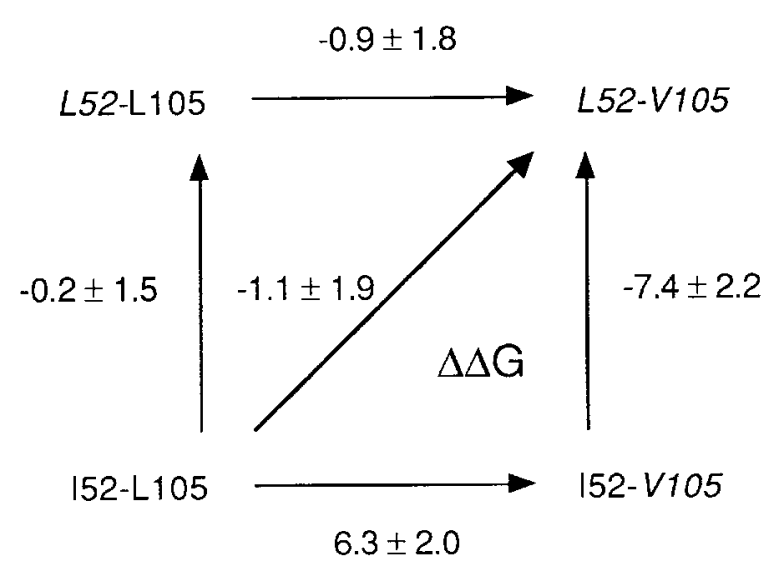

Figure 4. Thermodynamic cycles comparing the effects of single and double mutations on the unfolding of $\operatorname{TyrRS}(\Delta 1)$ by urea in the spectrofluorometry experiments. The mutant residues are indicated in italic type. The values of $\Delta \Delta G_{1}\left(\mathrm{H}_{2} \mathrm{O}\right), \Delta \Delta G_{2}\left(\mathrm{H}_{2} \mathrm{O}\right)$ and $\Delta \Delta G\left(\mathrm{H}_{2} \mathrm{O}\right)$ and the associated SE (kcal mol ${ }^{-1}$ ) were calculated from the values of $\Delta G_{1}\left(\mathrm{H}_{2} \mathrm{O}\right), \Delta G_{2}\left(\mathrm{H}_{2} \mathrm{O}\right)$ and $\Delta G\left(\mathrm{H}_{2} \mathrm{O}\right)$ in Table 4, by using equations (17), (18) and (21) in Materials and Methods.

double mutant thermodynamic cycles. For the association between the two subunits of $\operatorname{TyrRS}(\Delta 1)$, mutation I52L had a destabilizing effect both in the context of the wild-type $\operatorname{TyrRS}(\Delta 1)$ and in the context of the L105V mutant, with
$\Delta \Delta G_{1}\left(\mathrm{H}_{2} \mathrm{O}\right)=2.4( \pm 0.2)$ and $1.4( \pm 0.4) \mathrm{kcal} \mathrm{mol}^{-1}$, respectively. In contrast, mutation $\mathrm{L} 105 \mathrm{~V}$ was nearly neutral both in the context of the wild-type and in the context of the I52L mutant. For the unfolding of the monomeric intermediate, mutation L105V was strongly destabilizing in the context of the wild-type, with $\Delta \Delta G_{2}\left(\mathrm{H}_{2} \mathrm{O}\right)=2.8( \pm 1.0) \mathrm{kcal} \mathrm{mol}^{-1}$, and practically neutral in the context of the I52L mutant. Mutation I52L was slightly stabilizing in the context of the wild-type and strongly stabilizing in the context of the L105V mutant with $\Delta \Delta G_{2}\left(\mathrm{H}_{2} \mathrm{O}\right)=-4.4( \pm 1.1) \mathrm{kcal} \mathrm{mol}^{-1}$. For the total stability of the dimer, mutation L105V was again strongly destabilizing in the context of the wildtype, with $\Delta \Delta G\left(\mathrm{H}_{2} \mathrm{O}\right)=6.3( \pm 2.0) \mathrm{kcal} \mathrm{mol}^{-1}$, and neutral in the context of the I52L mutant. Mutation I52L was neutral in the context of the wild-type and strongly stabilizing in the context of the L105V mutant, with $\Delta \Delta \mathrm{G}\left(\mathrm{H}_{2} \mathrm{O}\right)=-7.4( \pm 2.2) \mathrm{kcal} \mathrm{mol}^{-1}$. From the free energies of unfolding and equation (20) (Materials and Methods), we also calculated the coupling energies between mutations I52L and L105V: $\Delta \Delta G_{1, \mathrm{~B}}\left(\mathrm{H}_{2} \mathrm{O}\right)=-1.0( \pm 0.5) \mathrm{kcal}$ $\mathrm{mol}^{-1} ; \quad \Delta \Delta G_{2, \mathrm{~B}}\left(\mathrm{H}_{2} \mathrm{O}\right)=-3.1( \pm 1.3) \mathrm{kcal} \mathrm{mol}^{-1}$ and $\Delta \Delta G_{\mathrm{B}}\left(\mathrm{H}_{2} \mathrm{O}\right) \stackrel{2, \mathrm{~B}}{=}-7.2( \pm 2.7) \mathrm{kcal} \mathrm{mol}^{-1}$. These coupling energies showed that residues Ile52 and Leu105 made an interaction which was energetically significant. Their comparison with the variations of free energy of unfolding due to mutations I52L and L105V (Figure 4), as described (Mildvan et al., 1992), confirmed that they had non-additive, compensatory effects.

\section{Unfolding by urea, monitored by fast-size exclusion chromatography}

Previously, we have studied the behaviour of the wild-type $\operatorname{TyrRS}(\Delta 1)$ in experiments of fast size-exclusion chromatography (fast-SEC) at different concentrations of urea. We have observed that $\operatorname{TyrRS}(\Delta 1)$ is eluted from the column of chromatography as a single peak of protein, whatever the concentration of urea. We have used the partition coefficient $K_{\mathrm{av}}$ of this single peak, which is a decreasing function of the mean Stokes radius $R_{\mathrm{S}}$, to monitor the unfolding of $\operatorname{TyrRS}(\Delta 1)$. The corresponding profile of unfolding has shown the following conformational transitions when the concentration of urea increases. The dimer of TyrRS( $\Delta 1)$ swells progressively between 0 and $4 \mathrm{M}$ urea, the two subunits dissociate cooperatively into a compact monomeric intermediate between 4.5 and $5.5 \mathrm{M}$ urea, the monomeric intermediate unfolds cooperatively between 5.5 and $6.5 \mathrm{M}$ urea, finally the unfolded polypeptide continues to stretch (Park \& Bedouelle, 1998; see Figure 5). Here, we performed identical experiments for the derivatives of $\operatorname{TyrRS}(\Delta 1)$ that carried the single mutations I52L or L105V, or the double mutation I52L-L105V. Each mutant derivative of TyrRS( $\Delta 1)$ was eluted from the chromatography column as a single peak of protein (with the restrictions below). 
The profiles of unfolding by urea for the mutants were similar to that for the wild-type, except that the maximal value of $K_{\mathrm{av}}$ was higher and the transitions were shifted towards lower concentrations of urea (Figure 5). Thus, all the mutants unfolded through a monomeric intermediate, as the wildtype, and the mutations were destabilizing. The higher maximal values of $K_{\mathrm{av}}$ for the mutants were compatible with higher molar fractions of the monomeric intermediate. We observed that a low percentage of the protein materials eluted with the void volume for the L105V single mutant and for the I52L-L105V double mutant in $5.5 \mathrm{M}$ urea. Moreover, the major protein peak was broader for the I52L-L105V double mutant than for the other derivatives of $\operatorname{TyrRS}(\Delta 1)$ in $5.5 \mathrm{M}$ urea.

To compare quantitatively the profiles of unfolding, induced with urea and monitored by fast-SEC, for the wild-type $\operatorname{TyrRS}(\Delta 1)$ and for its mutant derivatives, we fitted equation $\left(16^{\prime}\right)$ to each of them. Equation $\left(16^{\prime}\right)$ links the mean $K_{\mathrm{av}}$ of the different conformational states of a dimeric protein and the concentration of urea, for a mechanism of unfolding with a monomeric intermediate state (Materials and Methods). The values of the thermodynamic parameters that were deduced from these fittings, showed the following relative effects of the mutations on the stability of $\operatorname{TyrRS}(\Delta 1)$ (Table 5). The single mutation I52L and the double mutation
I52L-L105V destabilized the association between the subunits more than $\operatorname{L105V}\left(\Delta \Delta G_{1}\left(\mathrm{H}_{2} \mathrm{O}\right)=8,10\right.$ and $1 \mathrm{kcal} \mathrm{mol}^{-1}$, respectively). Mutation L105V destabilized the monomeric intermediate more than I52L-L105V, and I52L stabilized this intermediate $\left(\Delta \Delta G_{2}\left(\mathrm{H}_{2} \mathrm{O}\right)=8,6\right.$ and $-1 \mathrm{kcal} \mathrm{mol}^{-1}$, respectively). The absolute values of the thermodynamic parameters that we deduced from the experiments of fast-SEC, cannot be compared directly with those that we deduced from the experiments of spectrofluorometry, for reasons explained in the Discussion. It was nevertheless satisfying to observe that the relative effects of the mutations on the unfolding transitions were correlated in the two types of experiments, if one excepts the effect of I52L-L105V on the stability of the monomeric intermediate (Table 5 and Figure 4). This exception could be linked to the broader form of the chromatography peak for the I52L-L105V mutant at the critical concentration of $5.5 \mathrm{M}$ urea (see above).

\section{Discussion}

\section{Involvement of residues Thr51, Ile52 and Leu105 in the stability of $\operatorname{TyRSS}(\Delta 1)$}

We found that the single mutations T51P, I52L and L105V, and the double mutation I52L-L105V
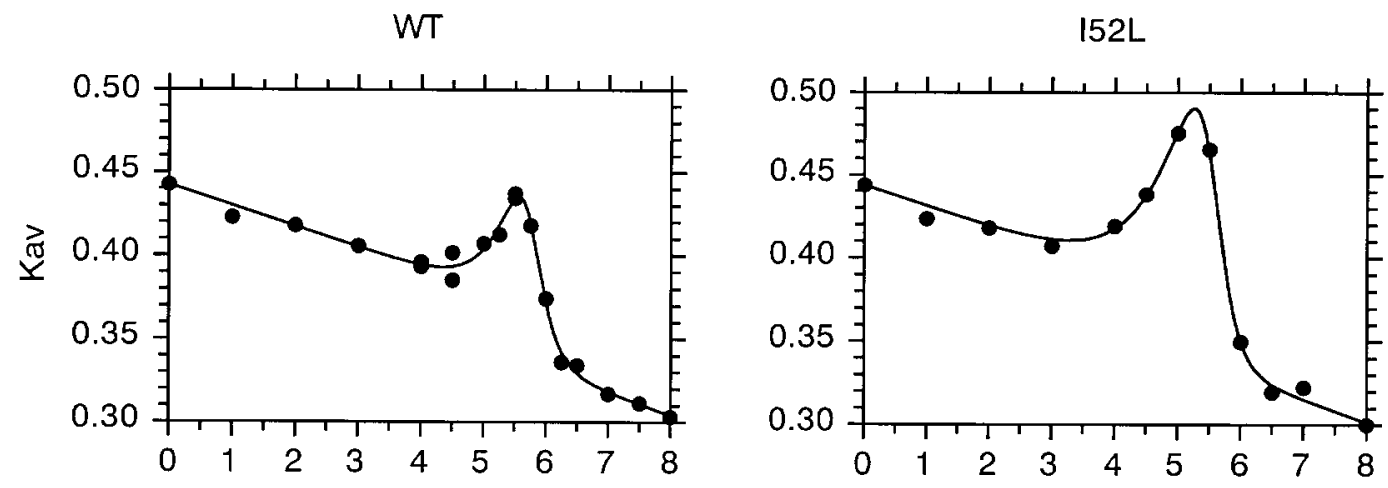

L105V

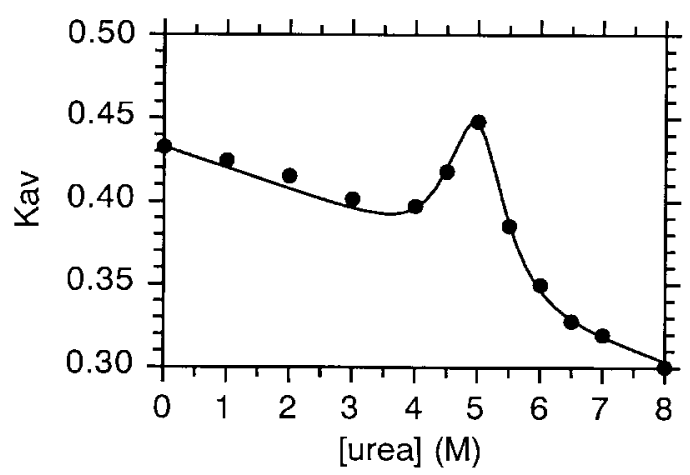

152L-L105V

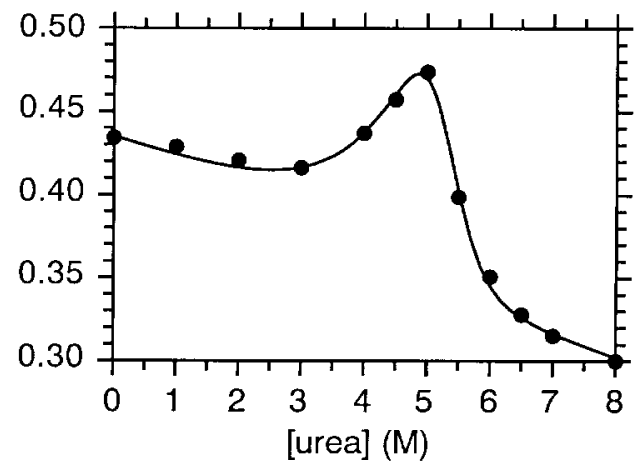

Figure 5. Unfolding of TyrRS $(\Delta 1)$ and its mutant derivatives by urea, as monitored by their mean partition coefficient $K_{\mathrm{av}}$ in fast size-exclusion chromatography experiments. The injected sample $(100 \mu \mathrm{l})$ contained the purified synthetase at a concentration of $100 \mu \mathrm{g} / \mathrm{ml}(1.4 \mu \mathrm{M})$. The continuous line was obtained by fitting equation $\left(16^{\prime}\right)$ to the experimental data. See Materials and Methods for details. 
Table 5. Parameters for the unfolding of $\operatorname{TyrRS}(\Delta 1)$ and its mutant derivatives by urea, as monitored by fast size-exclusion chromatography

\begin{tabular}{lccc}
\hline Parameter & I52L & L105V & I52L-L105V \\
\hline$\Delta m_{1}\left(\mathrm{kcal} \mathrm{mol}^{-1} \mathrm{M}^{-1}\right)$ & 1.1 & -0.13 & 1.7 \\
$\Delta \Delta G_{1}\left(\mathrm{H}_{2} \mathrm{O}\right)\left(\mathrm{kcal} \mathrm{mol}^{-1}\right)$ & 7.7 & 1.4 & 9.9 \\
$\Delta m_{2}\left(\mathrm{kcal} \mathrm{mol}^{-1} \mathrm{M}^{-1}\right)$ & -0.28 & 1.2 & 0.78 \\
$\Delta \Delta G_{2}\left(\mathrm{H}_{2} \mathrm{O}\right)\left(\mathrm{kcal} \mathrm{mol}^{-1}\right)$ & -1.4 & 8.0 & 5.7 \\
\hline
\end{tabular}

The unfolding of the synthetases was monitored by their mean partition coefficient $K_{\mathrm{av}}$ in experiments of fast-SEC, as described in the legend to Figure 5. The notations are the same as in Table 4. The thermodynamic parameters $m_{1}, m_{2}$, $\Delta G_{1}\left(\mathrm{H}_{2} \mathrm{O}\right)$ and $\Delta G_{2}\left(\mathrm{H}_{2} \mathrm{O}\right)$ were obtained by fitting equation $\left(16^{\prime}\right)$ to the experimental data and their variations were calculated by equation (17). The values for the wild-type were the following: $\quad m_{1}(\mathrm{wt})=2.93 \mathrm{kcal} \mathrm{mol}^{-1} \mathrm{M}^{-1} ; \quad m_{2}(\mathrm{wt})=2.64 \mathrm{kcal}$ $\mathrm{mol}^{-1} \mathrm{M}^{-1} ; \quad \Delta G_{1}\left(\mathrm{H}_{2} \mathrm{O}, \quad\right.$ wt $)=26.2 \mathrm{kcal} \mathrm{mol}^{-1} ; \quad \Delta G_{2}\left(\mathrm{H}_{2} \mathrm{O}\right.$, $\mathrm{wt})=14.3 \mathrm{kcal} \mathrm{mol}^{-1}$.

decreased the kinetic stability of $\operatorname{TyrRS}(\Delta 1)$ in the experiments of thermal precipitation. L105V also decreased the global thermodynamic stability of $\operatorname{TyrRS}(\Delta 1)$ and the stability of its monomeric intermediate of unfolding, measured in equilibrium conditions. T51P, I52L and I52L-L105V had no detectable effect on the global thermodynamic stability of $\operatorname{TyrRS}(\Delta 1)$ but they decreased the stability of the association between its subunits. As the monomer of $\operatorname{TyrRS}(\Delta 1)$ is inactive, a lower stability of the association between the subunits implied a lower functional stability (Bedouelle \& Winter, 1986; Carter et al., 1986). Thus, three residues of the dense cluster under study, Thr51, Ile52 and Leu105, were involved in the stability of $\operatorname{TyrRS}(\Delta 1)$. Moreover, the I52L-L105V double mutant had lower kinetic and functional stabilities than the wild-type, even though its global thermodynamic stability remained identical to that of the wild-type.

In the wild-type context, mutation I52L decreased the thermodynamic stability of the association between the subunits of $\operatorname{TyrRS}(\Delta 1)$ whereas L105V was nearly neutral for this stability. Mutation L105V decreased the stability of the monomeric intermediate of unfolding whereas I52L was nearly neutral for this stability. Thus, our results showed that mutations I52L and L105V affected the thermodynamic stabilities of different folding states of TyrRS( $\Delta 1)$. In the context of the L105V mutant, mutation I52L had opposite effects on the two partial equilibria of unfolding, i.e. it destabilized the association between the subunits and stabilized the monomeric intermediate, with $\Delta \Delta G_{1}\left(\mathrm{H}_{2} \mathrm{O}\right)=1.4 \quad( \pm 0.4) \mathrm{kcal} \quad \mathrm{mol}^{-1} \quad$ and $\Delta \Delta G_{2}\left(\mathrm{H}_{2} \mathrm{O}\right)=-4.4( \pm 1.1) \mathrm{kcal} \mathrm{mol}^{-1}$. Thus, in the context of the $\mathrm{L} 105 \mathrm{~V}$ mutant, the interactions between the side-chain of Ile52 and the remainder of the protein seemed to link the two partial unfolding events $\mathrm{N}_{2} \Longleftrightarrow 2 \mathrm{I}$ and $\mathrm{I} \Longleftrightarrow \mathrm{U}$, and the two corresponding stabilities, $\Delta G_{1}\left(\mathrm{H}_{2} \mathrm{O}\right)$ and $\Delta G_{2}\left(\mathrm{H}_{2} \mathrm{O}\right)$.

\section{Long range destabilizing effect of $152 \mathrm{~L}$}

Mutation I52L destabilized the association between the subunits of $\operatorname{TyrRS}(\Delta 1)$ strongly and T51P destabilized it significantly. These destabilizing effects were surprising because residues Ile52 and Thr51 are more than $20 \AA$ away from the interface between the subunits (Brick \& Blow, 1987). However, they can be rationalized if one notes that Thr51 belongs to the binding site of ATP, that Ile52 is close to it, and that TyrRS is an allosteric enzyme (Fersht, 1987). In particular, the crystal structure of the mutant TyrRS(C35G) has shown a structural coupling between Ile52 and residue Cys35, which belongs to the binding site of ATP (Fothergill \& Fersht, 1991). The mutant TyrRS(K233A) shows a positive cooperativity for the binding of ATP, which implies a communication between its two distant binding sites across the dimerization interface (First \& Fersht, 1993). The molecule of TyrRS is symmetrical, binds two molecules of tyrosine and forms two molecules of tyrosyl-adenylate in the crystals whereas it is asymmetrical, binds only one molecule of tyrosine and forms only one molecule of tyrosyl-adenylate in solution (Brick et al., 1989; Ward \& Fersht, 1988). The properties of mutations I52L and T51P suggest that it will be possible to study directly and quantitatively the effects of mutations, located around the active sites, on the interaction between the two subunits of $\operatorname{TyrRS}(\Delta 1)$, and thus the transfer of information through their interface.

\section{Coupling between mutations I52L and L105V, and context effect}

We found that the effect of mutation M55L on the kinetic stability of $\operatorname{TyrRS}(\Delta 1)$ was not coupled to those of mutations I52L, L105V or I52L-L105V in the experiments of thermal precipitation. These results suggested that the evolution of residue Met55 occurred independently of the other residues of the dense cluster under study. In contrast, mutations I52L and L105V had coupled effects, for the kinetic stability of $\operatorname{TyrRS}(\Delta 1)$ with $\Delta \Delta G_{\mathrm{B}}(\mathrm{I} 52 \mathrm{~L}$, $\mathrm{L} 105 \mathrm{~V})=-1.0( \pm 0.1) \mathrm{kcal} \mathrm{mol}^{-1}$, for the thermodynamic stability of the association between the subunits with $\Delta \Delta G_{1, \mathrm{~B}}\left(\mathrm{H}_{2} \mathrm{O}\right)=-1.0 \quad( \pm 0.5) \mathrm{kcal}$ $\mathrm{mol}^{-1}$, for the stability of the monomeric intermediate with $\Delta \Delta G_{2, \mathrm{~B}}\left(\mathrm{H}_{2} \mathrm{O}\right)=-3.1 \quad( \pm 1.3) \mathrm{kcal}$ $\mathrm{mol}^{-1}$, and for the total stability of $\operatorname{TyrRS}(\Delta 1)$ with $\Delta \Delta G_{\mathrm{B}}\left(\mathrm{H}_{2} \mathrm{O}\right)=-7.2( \pm 2.7) \mathrm{kcal} \mathrm{mol}^{-1}$. The values of the coupling energies showed that the tertiary contact between the side-chains of Ile52 and Leu105, as it appears in the crystal structure, had some energetic importance for the stability of $\operatorname{TyrRS}(\Delta 1)$. They also showed that the effect of each of mutations I52L and L105V depended on the side-chain in the other position. Such dependence on the structural context for mutations in the core of proteins has already been described (Zhang et al., 1996). 


\section{Evolutionary implications}

The observed couplings between the effects of mutations I52L and L105V had the following implications for the possible evolutionary pathways that led from the wild-type to the I52LL105V double mutant, through one or the other of the two single mutants (Figure 4). For the stability of the association between the subunits, the two pathways comprised a destabilizing step, corresponding to mutation $152 \mathrm{~L}$, and a nearly neutral step, corresponding to L105V. As a result, the association between the subunits of the double mutant was less stable than that of the wild-type $\operatorname{TyrRS}(\Delta 1)$. It will be interesting to test whether this difference of stability exists between the wildtype TyrRSs from B. stearothermophilus and E. coli or whether it is compensated by residue changes outside of the dense cluster under study. For the stability of the monomeric intermediate or the total stability of $\operatorname{TyrRS}(\Delta 1)$, one of the pathways, going through the I52L single mutant, comprised only neutral or slightly stabilizing steps, whereas the other pathway, going through the L105V single mutant, comprised a strongly destabilizing step followed by a strongly compensatory step. As a result, the double mutation had a neutral or slightly stabilizing effect. Thus, mutation I52L allowed the deleterious mutation L105V along the first pathway and compensated it along the second one. Such a behaviour has been predicted by Hardies \& Garvin (1991) in their theory of the context effect, that integrates both a neutral drift and a positive selection. Our results on mutations I52L and $\mathrm{L} 105 \mathrm{~V}$ are thus compatible with the concepts of a context effect and of a preferential pathway of evolution.

\section{Comparison between the fast-SEC and fluorescence experiments}

The unfolding profiles of $\operatorname{TyrRS}(\Delta 1)$ and of its mutant derivatives by urea, monitored by fastSEC, showed directly that each mutant derivative of $\operatorname{TyrRS}(\Delta 1)$ unfolded through a compact monomeric intermediate state, as the wild-type. This result validated our use of thermodynamic models of unfolding that comprised a monomeric intermediate, to compare the stabilities of $\operatorname{TyrRS}(\Delta 1)$ and of its mutant derivatives quantitatively. The thermodynamic models that we used to analyse the unfolding profiles were identical for the fluorometric and fast-SEC experiments, except that the relation that linked the global intensity of fluorescence to the molar intensities of the conformational states in equilibrium, and the relation that linked the mean $K_{\mathrm{av}}$ to the $K_{\mathrm{av}} \mathrm{s}$ of the states in equilibrium were different (equations (9) and $\left(10^{\prime}\right)$ ). We found that the protein was eluted from the column as a single peak of chromatography in the fast-SEC experiments, whatever the concentration of urea (with the restriction mentioned in Results). This observation showed that the different confor- mational states of each mutant were in rapid exchange, when compared to the length of the chromatographic run, and thus that they were in quasi-equilibrium. The total concentration $C$ of $\operatorname{TyrRS}(\Delta 1)$ decreased progressively during the chromatographic runs and therefore was not strictly constant, as assumed in our equations. This approximation led to larger values of the thermodynamic parameters in the fast-SEC experiments than in the fluorometric experiments. However, the fast-SEC experiments were done in exactly the same conditions and the approximations were the same for the wild-type $\operatorname{TyrRS}(\Delta 1)$ as for its mutants, so that our thermodynamic analysis of these experiments was useful to compare semiquantitatively the effects of the mutations on the stability of $\operatorname{TyrRS}(\Delta 1)$. In fact, the effects of the mutations on the stability of $\operatorname{TyrRS}(\Delta 1)$ that we deduced from the fast-SEC experiments, were indeed correlated with those that we deduced from the more quantitative spectrofluorometric experiments (Table 5 and Figure 4).

\section{Comparison between the denaturations by temperature and urea}

Several structural events could lead to a decrease in the fluorescence intensity of $\operatorname{TyrRS}(\Delta 1)$ in the experiments of thermal denaturation. The subunits of $\operatorname{TyrRS}(\Delta 1)$ could dissociate, the protein could unfold, or it could precipitate and be eliminated during the centrifugation step. We found that the fluorescence intensity of the soluble protein fraction could decrease down to less than $1 \%$ of its initial value during the kinetics of thermal denaturation for some of the $\operatorname{TyrRS}(\Delta 1)$ derivatives (Figure 2). The fluorescence intensity of the unfolded state is equal to $15 \%$ that of one subunit of the native dimer for $\operatorname{TyrRS}(\Delta 1)$ (Park \& Bedouelle, 1998). Therefore, the decrease of the fluorescence signal in the experiments of thermal denaturation was necessarily due to an irreversible precipitation. This conclusion is compatible with the results of similar experiments in which the amount of protein in the soluble fraction was analysed by gel electrophoresis (Guez-Ivanier \& Bedouelle, 1996). We observed that the fluorescence intensity of the soluble fraction was a simple exponential function of time during the thermal denaturation. Therefore the thermal denaturation was a first order reaction. This observation indicated that the limiting step was unimolecular and thus an unfolding event preceding aggregation, because aggregation is a multimolecular reaction.

The values of $\Delta \Delta G^{*}$ in the experiments of thermal precipitation enabled us to $\operatorname{rank} \operatorname{TyrRS}(\Delta 1)$ and its mutant derivatives in the following order: wild-type $<$ T51P $<$ I52L $<$ I52L-L105V $<$ L105V (Table 2). The maximum, for each mutation, of $\Delta \Delta G_{1}\left(\mathrm{H}_{2} \mathrm{O}\right)$ and $\Delta \Delta G_{2}\left(\mathrm{H}_{2} \mathrm{O}\right)$ in the experiments of unfolding by urea, monitored by spectrofluorometry, gave about the same order: $\Delta \Delta G_{1}\left(\mathrm{H}_{2} \mathrm{O}\right)=0.8$ 
$( \pm 0.2) \mathrm{kcal} \mathrm{mol}^{-1}$ for T51P, $2.1( \pm 0.2)$ for I52LL105V, $2.4( \pm 0.2)$ for I52L, and $\Delta \Delta G_{2}\left(\mathrm{H}_{2} \mathrm{O}\right)=2.8$ $( \pm 1) \mathrm{kcal} \mathrm{mol}^{-1}$ for L105V. The comparison of these two orders, based either on $\Delta \Delta G^{\ddagger}$ or on $\max \left(\Delta \Delta G_{1}\left(\mathrm{H}_{2} \mathrm{O}\right), \Delta \Delta G_{2}\left(\mathrm{H}_{2} \mathrm{O}\right)\right)$, suggested that both a destabilization of the association between the subunits and a destabilization of the monomeric intermediate led to a correlated decrease of the kinetic stability. In addition, the effects of mutations I52L and L105V were dependent (nonadditive) in both types of experiments. Thus, the experiments of thermal precipitation seem a reliable and simple test to screen mutations that affect the stability of TyrRS. The observation that L105V both destabilized the monomeric intermediate and increased the rate of thermal precipitation, suggested that the conformational state of $\operatorname{TyrRS}(\Delta 1)$ that precipitated, came after the monomeric intermediate on the pathway of unfolding.

\section{Further discussion and conclusions}

We found that mutations I52L, M55L and L105V did not affect the pyrophosphate exchange activity of $\operatorname{TyrRS}(\Delta 1)$ significantly. Whether they affect or not the charging of tRNA ${ }^{\mathrm{Tyr}}$ could be tested by introducing them into the full-length TyrRS. We found that the improvement of the TyrRS activity by mutation T51P was realized at the expense of a slight loss of conformational stability. This loss of stability occurred without structural changes other than local, as shown by the crystal structure of the mutant TyrRS(T51P) (Brown et al., 1987). Mutations I52L and L105V decreased the kinetic stability of TyrRS from B. stearothermophilus at $68.5^{\circ} \mathrm{C}$, which is the temperature of its natural habitat; therefore they may have a physiological significance. The single mutation I52L and the double mutation I52L-L105V destabilized the association between the subunits of TyrRS; L105V destabilized the monomeric intermediate state. Therefore, three residues of the dense cluster under study, Thr51, Ile52 and Leu105, were involved in the stability of TyrRS and our results confirmed that dense clusters could indeed be involved in the stability and folding of proteins. They also showed that a dense cluster, located close to the active site and far from the subunit interface, could destabilize the association between the subunits. Thus, the methods that we developed here, should allow us to directly study the transmission of information between the active sites across the subunit interface. The effects of mutations I52L and L105V on the stability of TyrRS were not additive. Thus, the tertiary interactions between the side-chains of residues Ile52 and Leu105, and therefore between helices $\mathrm{H} 3$ and H5, were important for the stability of TyrRS. Our results showed that the effects of mutations I52L and L105V on the stability of TyrRS depended on their structural context. In particular, we showed that there could be a preferential evolutionary pathway and that a first mutation could allow or compensate a second mutation, depending on the pathway. Our results were thus compatible with the theory of the context effect (Hardies \& Garvin, 1991). They also showed that the effects of I52L and L105V depended on the folding state of $\operatorname{TyrRS}(\Delta 1)$, either the native dimer or the monomeric intermediate of unfolding. For example, mutation I52L had a neutral effect on the global stability of TyrRS even though it destabilized the association between the subunits. Thus, dissecting the pathway of unfolding into its different states can give essential information on the effects of mutations.

\section{Materials and Methods}

\section{Dense clusters of residues}

We used the CLUSPROT program (Heringa \& Argos, 1991; Heringa et al., 1995; http://www.embl-heidelberg.de/argos/clusprot) to identify the dense clusters of residues that include Ile52 and Leu105 in TyrRS from B. stearothermophilus. We used the atomic coordinates of entries 2ts1, which corresponds to one of the two symmetrical subunits of the full-length TyrRS, and 4ts1, which corresponds to the truncated dimer $\operatorname{TyrRS}(\Delta 1)$, in the Protein Data Bank (PDB). CLUSPROT delineates spatial clusters in protein structures on the basis of atomic contacts between residues. We performed the calculations only on the side-chains of the amino acid residues, with local normalization values. The dense neighbour threshold, $8.0 \AA$, and the other parameters were the default ones. The structures of the dense clusters were analysed with the WHAT IF program (http:/ / www.sander.embl-heidelberg.de/whatif/).

\section{Genetic techniques}

Strains RZ1032 (Kunkel et al., 1987), TG2, and phage $\operatorname{M13-BY}(\Delta 1)$ (Waye et al., 1983; Guez-Ivanier \& Bedouelle, 1996) have been described. We chose codons that are frequent in the abundant proteins of E. coli to replace codons 51, 52, 55 and 105 in the tyrS gene of B. stearothermophilus: Leu, CTG; Pro, CCG; Val, GTT. The mutations were intoduced into phage $\operatorname{M13BY}(\Delta 1)$ by a published method of mutagenesis (Kunkel et al., 1987). The template DNA was produced in strain RZ1032 (dut, ung) and the mutated DNA was transfected into strain TG2. We checked the full sequence of the mutant tyrS genes.

\section{Proteins and general conditions}

$\operatorname{TyrRS}(\Delta 1)$ and its mutant derivatives were overexpressed from phage $\operatorname{M13-BY}(\Delta 1)$ and its derivatives, and purified as described (Vidal-Cros \& Bedouelle, 1992; Guez-Ivanier \& Bedouelle, 1996). The purified proteins were more than $95 \%$ pure as judged by gel electrophoresis. They were stored at $-70^{\circ} \mathrm{C}$ in $20 \mathrm{mM}$ Tris- $\mathrm{HCl}$ ( $\mathrm{pH} 7.78)$ and $5 \mathrm{mM}$ 2-mercaptoethanol. The concentration of protein in the purified samples was measured with the Bio-Rad protein assay kit and bovine serum albumin as a standard. The molecular mass of $\operatorname{TyrRS}(\Delta 1)$ and its mutant derivatives was taken as $36,324 \mathrm{Da} / \mathrm{sub}-$ unit (Waye et al., 1983). All the experiments were performed at $25^{\circ} \mathrm{C}$. The active site titration and the pyrophosphate exchange assay were performed as described (Wilkinson et al., 1983; Vidal-Cros \& 
Bedouelle, 1992). The reactions of unfolding and refolding in the presence of urea were performed and brought to equilibrium as described (Guez-Ivanier \& Bedouelle, 1996; Park and Bedouelle, 1998). The concentration of urea in the reaction mixture was measured with a refractometer and a precision of $0.01 \mathrm{M}$ after the completion of each experiment. Ultrapure urea was purchased from ICN and used directly as provided.

\section{Irreversible thermal precipitation}

$\operatorname{TyrRS}(\Delta 1)$ and its mutant derivatives $(100 \mu \mathrm{g} / \mathrm{ml}$ in buffer A) were heated at $68.5^{\circ} \mathrm{C}$ during increasing times. Several identical samples (100 $\mu \mathrm{l}$ in Eppendorf tubes) were prepared for each synthetase and heated simultaneously in a water bath. At several time points, one tube was withdrawn from the water bath, rapidly put on ice and held there for at least 15 minutes. At the end of the kinetics, the protein precipitate was removed by centrifugation. Each soluble fraction was diluted ten times in buffer $\mathrm{A}$ and its intensity of light emission was measured as described below. Buffer A was $50 \mathrm{mM}$ Trishydrochloride ( $\mathrm{pH} 7.78), 0.1 \mathrm{mM}$ phenyl-methylsulphonide fluoride, $5 \mathrm{mM}$ 2-mercaptoethanol.

\section{Intrinsic fluorescence experiments}

The intensity of fluorescence emission was measured with a Perkin-Elmer LS-5B spectrofluorometer at $25^{\circ} \mathrm{C}$. The samples were excited at $278 \mathrm{~nm}$ with a slit width equal to $2.5 \mathrm{~nm}$. The signal of emission was averaged during 17 seconds. The signal for the protein was corrected by substraction of the signal for the solvent alone. The wavelength of light emission and the slit width were $340 \mathrm{~nm}$ and $2.5 \mathrm{~nm}$, respectively, for the experiments of irreversible thermal precipitation. They were $330 \mathrm{~nm}$ and $5 \mathrm{~nm}$, respectively, for the experiments of unfolding by urea.

\section{Size-exclusion chromatography}

The hydrodynamic properties of $\operatorname{TyrRS}(\Delta 1)$ and its mutant derivatives and their variations with the concentration of urea were measured by size-exclusion chromatography through a Superdex 200 HR 10/30 column (Pharmacia-Biotech) as described (Park \& Bedouelle, 1998). The effluent was continuously monitored by $A_{280 \mathrm{~nm}}$. An aliquot $(100 \mu \mathrm{l})$ of an unfolding reaction, containing $100 \mu \mathrm{g} / \mathrm{ml}$ purified protein, was injected at the top of the column. The column was eluted with the same buffer that was used in the unfolding reaction. The measures were expressed using the partition coefficient $K_{\mathrm{av}}=\left(V_{\mathrm{e}}-V_{0}\right) /\left(V_{\mathrm{t}}-V_{0}\right)$, where $V_{\mathrm{e}}$ is the elution volume corresponding to the maximum of the protein elution peak, and $V_{\mathrm{t}}$ and $V_{0}$ are the total volume and the void volume of the column, respectively. All the chromatographic runs were performed with a flow rate of $0.3 \mathrm{ml} / \mathrm{min}$ and at room temperature $\left(22-26^{\circ} \mathrm{C}\right)$.

\section{Analysis of the thermal precipitation profiles}

The kinetics of thermal precipitation at $68.5^{\circ} \mathrm{C}$ were followed by the fluorescence intensity $Y$ of the soluble fraction, as described above. The rate constant of precipitation, $k$, was obtained by fitting equation:

$$
Y=a^{*} \exp \left(-k^{*} t\right)
$$

to the experimental values. The goodness of fitting was measured with the Pearson coefficient, $R_{\mathrm{P}}$. To draw Figure 2, the fluorescence intensities were normalized by the transformation $Y \rightarrow Y / a$. The free energy of activation for the reaction of thermal precipitation was deduced from the theory of the transition state by equation:

$$
\Delta G^{\star}=R T \ln \left(k_{\mathrm{B}} T / h\right)-R T \ln (k)
$$

where $R$ is the gas constant, $T$ is temperature $(341.66 \mathrm{~K})$, $k_{\mathrm{B}}$ is the Boltzmann constant and $h$ is the Plank constant.

\section{Analysis of the unfolding profiles, monitored by fluorescence intensity}

$\operatorname{TyrRS}(\Delta 1)$ unfolds through a monomeric intermediate according to the equilibria:

$$
\mathrm{N}_{2} \Leftrightarrow 2 \mathrm{I} \Leftrightarrow 2 \mathrm{U}
$$

where $\mathrm{N}_{2}$ is the native dimer, $\mathrm{I}$, the monomeric intermediate, and $U$, the unfolded state. In physiological conditions, the protein is almost entirely in its native form $\mathrm{N}_{2}$ and the concentrations of states I and $\mathrm{U}$ cannot be detected (Park \& Bedouelle, 1998). To study the equilibria of unfolding for this type of protein, one generally shifts them with a denaturing agent, like urea. For each concentration of denaturant, noted $x$, new equilibria form. One obtains an unfolding profile by measuring a global signal of the protein as a function of $x$. The following equations allow one to determine the concentration of each folding state, $N_{2}$, I and $U$, for each value of $x$.

Two equations are given by the law of mass action:

$$
K_{1}=[\mathrm{I}]^{2} /\left[\mathrm{N}_{2}\right] \text { and } K_{2}=[\mathrm{U}] /[\mathrm{I}]
$$

where $K_{1}$ and $K_{2}$ are equilibrium constants. A third equation is given by the law of mass conservation:

$$
\left[\mathrm{N}_{2}\right]+[\mathrm{I}] / 2+[\mathrm{U}] / 2=\mathrm{C}
$$

where $C$ is the total concentration $(M)$ of protein, expressed as dimer equivalent. The variation of free energy $\Delta G_{1}$ between states $N_{2}$ and $I$, and $\Delta G_{2}$ between states $I$ and $U$ are given by the equations:

$$
\Delta G_{1}=-R T \ln \left(K_{1}\right) \text { and } \Delta G_{2}=-R T \ln \left(K_{2}\right)
$$

where $R$ is the gas constant and $T$ is the temperature $(\mathrm{K})$. The equilibrium constant $K$ and the variation of free energy $\Delta G$ between states $\mathrm{N}_{2}$ and $2 \mathrm{U}$ are given by

$$
K=K_{1} K_{2}^{2} \text { and } \Delta G=\Delta G_{1}+2 \Delta G_{2} .
$$

By definition, $\Delta G_{1}$ is the stability of the association between the two subunits of the dimer, $\Delta G_{2}$ is the stability of the intermediate monomeric state and $\Delta G$ is the global stability of the dimer. Generally, one assumes that the variation of free energy between two conformational states is a linear function of $x$ (Myers et al., 1995):

$$
\Delta G_{1}=\Delta G_{1}\left(\mathrm{H}_{2} \mathrm{O}\right)-m_{1} x \text { and } \Delta G_{2}=\Delta G_{2}\left(\mathrm{H}_{2} \mathrm{O}\right)-m_{2} x
$$

If spectrometric techniques are used to monitor the unfolding equilibria of equation (3) and if $Y^{\prime}$ is the global signal of the unfolding mixture, the law of additivity of the signals applies:

$$
Y^{\prime}=Y_{\mathrm{n}}\left[\mathrm{N}_{2}\right]+Y_{\mathrm{i}}[\mathrm{I}]+Y_{\mathrm{u}}[\mathrm{U}]+Y_{\mathrm{d}} x
$$

where $Y_{\mathrm{n}}, Y_{\mathrm{i}}, Y_{\mathrm{u}}$ and $Y_{\mathrm{d}}$ are the molar signals $\left(\mathrm{M}^{-1}\right)$ of states $\mathrm{N}_{2}, \mathrm{I}, \mathrm{U}$ and of the denaturant respectively. The signal of the denaturant alone is generally measured in a 
separate experiment, and only the protein signal $Y$ is considered:

$$
Y=Y^{\prime}-Y_{\mathrm{d}} x \text {. }
$$

Experimentally, one observes that $Y_{\mathrm{n}}$ and $Y_{\mathrm{u}}$ are linear functions of $x$ at low and high concentrations of denaturant, respectively (Pace, 1986). $Y_{\mathrm{i}}$ is usually considered as constant since the intermediate exists in detectable concentration only in a short interval of $x$.

$$
Y_{\mathrm{n}}=y_{\mathrm{n}}+m_{\mathrm{n}} x ; \quad Y_{\mathrm{u}}=y_{\mathrm{u}}+m_{\mathrm{u}} x ; \quad Y_{\mathrm{i}}=\text { constant. }
$$

Generally, it is more convenient to reason on molar fractions:

$$
f_{\mathrm{n}}=\left[\mathrm{N}_{2}\right] / C, f_{\mathrm{u}}=[\mathrm{U}] / 2 \mathrm{C} \text { and } f_{\mathrm{i}}=[\mathrm{I}] / 2 \mathrm{C} .
$$

These molar fractions are chosen such that $f_{\mathrm{n}}+f_{\mathrm{i}}+f_{\mathrm{u}}=1$ when the law of mass conservation is applied. The signal $Y$ of the protein can then be expressed as a function of $x$ and of a number of parameters, ten in the present case. Two of these parameters are known: $C$, the total concentration of protein, and $y_{n}$, the molar signal of the native state in the absence of denaturant. Therefore, one is left with eight unknown parameters for eight independent equations $(4,5,8,9$ and 11). The solving equations are the following:

$$
\begin{gathered}
4 C f_{\mathrm{i}}^{2}+K_{1}\left(1+K_{2}\right) f_{\mathrm{i}}-K_{1}=0 \\
f_{\mathrm{n}}=1-\left(1+K_{2}\right) f_{\mathrm{i}} \\
f_{\mathrm{u}}=K_{2} f_{\mathrm{i}} \\
Y=C\left(Y_{\mathrm{n}}+\left(2 Y_{\mathrm{i}}-Y_{\mathrm{n}}+K_{2}\left(2 Y_{\mathrm{u}}-Y_{\mathrm{n}}\right)\right) f_{\mathrm{i}}\right)
\end{gathered}
$$

When the unfolding of $\operatorname{TyrRS}(\Delta 1)$ and its mutant derivatives, induced with urea, was monitored by their fluorescence intensity, we used equations (9) and (16). To facilitate the comparison of the unfolding profiles between the wild-type $\operatorname{TyrRS}(\Delta 1)$ and its mutant derivatives, we expressed the global protein signal $Y$ and the molar signals, specific of the different conformational states, as normalized parameters: i.e. we performed the transformations $Y \rightarrow Y / C^{*} y_{n}, \quad Y_{n} \rightarrow Y_{n} / y_{n}, \quad Y_{i} \rightarrow Y_{i} / y_{n}$ and $Y_{\mathrm{u}} \rightarrow Y_{\mathrm{u}} / y_{\mathrm{n}}$. To fit equation (16) to the unfolding profiles of the mutant enzymes, the starting parameters were taken equal to the fitting parameters of the wildtype enzyme (Park \& Bedouelle, 1998), and parameters $y_{\mathrm{n}}=1$ and $m_{\mathrm{n}}$ were kept constant.

\section{Analysis of the unfolding profiles, monitored by fast-SEC}

If fast-SEC is used to monitor the unfolding equilibria of equation (3), and if $Y$ is the mean partition coefficient (usually noted $K_{\mathrm{av}}$ ) of the protein, $Y$ is equal to the weighted average of the specific signals for the different conformational states of the protein (Ackers, 1970), so that equations (9) and (10) are replaced by

$$
Y\left(\left[\mathrm{~N}_{2}\right]+[\mathrm{I}]+[\mathrm{U}]\right)=Y_{\mathrm{n}}\left[\mathrm{N}_{2}\right]+Y_{\mathrm{i}}[\mathrm{I}]+Y_{\mathrm{u}}[\mathrm{U}]
$$

and equation (16) by:

$$
Y=\left(Y_{\mathrm{n}}+\left(2 Y_{\mathrm{i}}-Y_{\mathrm{n}}+K_{2}\left(2 Y_{\mathrm{u}}-Y_{\mathrm{n}}\right)\right) f_{\mathrm{i}}\right) /\left(1+\left(1+K_{2}\right) f_{\mathrm{i}}\right) \text {. }
$$

When the unfolding of $\operatorname{TyrRS}(\Delta 1)$ and its mutant derivatives, induced with urea, was monitored by their mean partition coefficient $K_{\mathrm{av}}$, we used equations $\left(10^{\prime}\right)$ and $\left(16^{\prime}\right)$. To fit equation $\left(16^{\prime}\right)$ to the unfolding profiles of the mutant enzymes, the starting parameters were taken equal to the fitting parameters of the wild-type (Park \& Bedouelle, 1998), except $y_{n}$ which was taken equal to the $K_{\mathrm{av}}$ of the mutant enzyme in the absence of urea. Parameters $y_{\mathrm{n}}, m_{\mathrm{n}}$ and $m_{\mathrm{u}}$ were kept constant during the fitting.

\section{Analyses of the unfolding energetics and coupling parameters}

If $P$ was the thermodynamic parameter under consideration, its variation when going from the wild-type (wt) to a mutant (mut) was calculated by:

$$
\Delta P(\text { wt }, \text { mut })=P(\text { wt })-P(\text { mut }) .
$$

Its variation when going from a first mutant $\left(\mathrm{mut}_{1}\right)$ to a second one $\left(\mathrm{mut}_{2}\right)$ was calculated by:

$$
\Delta P\left(\text { mut }_{1}, \text { mut }_{2}\right)=P\left(\text { mut }_{1}\right)-P\left(\text { mut }_{2}\right)
$$

$\Delta P_{\mathrm{A}}$, the effect of the less damaging mutation, mut $_{2}$, in the context of the more damaging mutation, mut $_{1}$, was calculated by:

$\Delta P_{\mathrm{A}}\left(\mathrm{mut}_{1}, \mathrm{mut}_{2}\right)=\Delta P\left(\mathrm{wt}, \mathrm{mut}_{1}-\mathrm{mut}_{2}\right)-\Delta P\left(\mathrm{wt}, \mathrm{mut}_{1}\right)$, or

$\Delta P_{\mathrm{A}}\left(\right.$ mut $_{1}$, mut $\left._{2}\right)=P\left(\right.$ mut $\left._{1}\right)-\mathrm{P}\left(\right.$ mut $_{1}-$ mut $\left._{2}\right)$

where mut $_{1}-$ mut $_{2}$ represents the double mutation. $\Delta P_{\mathrm{B}}$ the coupling parameter between mutations mut $_{1}$ and mut $_{2}$, was calculated by equation:

$$
\begin{aligned}
\Delta P_{\mathrm{B}}\left(\mathrm{mut}_{1}, \mathrm{mut}_{2}\right)= & \Delta P\left(\mathrm{wt}, \mathrm{mut}_{1}-\mathrm{mut}_{2}\right) \\
& -\Delta P\left(\mathrm{wt}, \mathrm{mut}_{1}\right)-\Delta P\left(\mathrm{wt}, \mathrm{mut}_{2}\right),
\end{aligned}
$$

or

$$
\begin{aligned}
\Delta P_{\mathrm{B}}\left(\mathrm{mut}_{1}, \mathrm{mut}_{2}\right)= & P\left(\text { mut }_{1}\right)+P\left(\text { mut }_{2}\right) \\
& -P\left(\text { mut }_{1}-\mathrm{mut}_{2}\right)-P(\mathrm{wt}) .
\end{aligned}
$$

If $a_{\mathrm{i}}(\mathrm{i}=1, \ldots, n)$ were values of measurements, the standard error (SE) on the sum of the $a_{\mathrm{i}}$ values was calculated from SE on the individual $a_{\mathrm{i}}$ values by the formula:

$$
\left[\operatorname{SE}\left(\Sigma_{i} \mathrm{a}_{i}\right)\right]^{2}=\Sigma_{i}\left[\operatorname{SE}\left(a_{\mathrm{i}}\right)\right]^{2} .
$$

\section{Acknowledgements}

We thank M. E. Goldberg for his constant interest.

\section{References}

Ackers, G. K. (1970). Analytical gel chromatography of proteins. Advan. Protein Chem. 24, 343-446.

Alber, T. (1989). Mutational effects on protein stability. Annu. Rev. Biochem. 58, 765-798.

Baldwin, E. P. \& Matthews, B. W. (1994). Core-packing constraints, hydrophobicity and protein design. Curr. Opin. Biotech. 5, 396-402.

Bedouelle, H. \& Winter, G. (1986). A model of synthetase/tRNA interaction as deduced by protein engineering. Nature, 320, 371-373. 
Brick, P. \& Blow, D. M. (1987). Crystal structure of a deletion mutant of a tyrosyl-tRNA synthetase complexed with tyrosine. J. Mol. Biol. 194, 287-297.

Brick, P., Bhat, T. N. \& Blow, D. M. (1989). Structure of tyrosyl-tRNA synthetase refined at $2.3 \AA$ resolution. Interaction of the enzyme with the tyrosyl adenylate intermediate. J. Mol. Biol. 208, 83-98.

Brown, K. A., Brick, P. \& Blow, D. M. (1987). Structure of a mutant of tyrosyl-tRNA synthetase with enhanced catalytic properties. Nature, 326, 416-418.

Carter, P., Bedouelle, H. \& Winter, G. (1986). Construction of heterodimer tyrosyl-tRNA synthetase shows tRNATyr interacts with both subunits. Proc. Natl Acad. Sci. USA, 83, 1189-1192.

Cheng, X., Gonzalez, M. L. \& Lee, J. C. (1993). Energetics of intersubunit and intrasubunit interactions of Escherichia coli adenosine cyclic 3',5'-phosphate receptor protein. Biochemistry, 32, 8130-8139.

Clark, A. C., Sinclair, J. F. \& Baldwin, T. O. (1993). Folding of bacterial luciferase involves a non-native heterodimeric intermediate in equilibrium with the native enzyme and the unfolded subunits. J. Biol. Chem. 268, 10773-10779.

Eftink, M. R., Helton, K. J., Beavers, A. \& Ramsay, G. D. (1994). The unfolding of trp aporepressor as a function of $\mathrm{pH}$ : evidence for an unfolding intermediate. Biochemistry, 33, 10220-10228.

Fersht, A. R. (1987). Dissection of the structure and activity of the tyrosyl-tRNA synthetase by sitedirected mutagenesis. Biochemistry, 26, 8031-8037.

Fersht, A. R. (1997). Nucleation mechanisms in protein folding. Curr. Opin. Struct. Biol. 7, 3-9.

Fersht, A. R. \& Serrano, L. (1993). Principles of protein stability derived from protein engineering experiments. Curr. Opin. Struct. Biol. 3, 75-83.

Fersht, A. R., Matouschek, A. \& Serrano, L. (1992). The folding of an enzyme. I. Theory of protein engineering, analysis of stability and pathway of protein folding. J. Mol. Biol. 224, 771-782.

First, E. A. \& Fersht, A. R. (1993). Mutation of lysine 233 to alanine introduces positive cooperativity into tyrosyl-tRNA synthetase. Biochemistry, 32, 13651-13657.

Fothergill, M. D. \& Fersht, A. R. (1991). Correlations between kinetic and X-ray analyses of engineered enzymes: crystal structures of mutants Cys $\rightarrow$ Gly35 and $\mathrm{Tyr} \rightarrow$ Phe-34 of tyrosyl-tRNA synthetase. Biochemistry, 30, 5157-5164.

Grimsley, J. K., Scholtz, J. M., Pace, C. N. \& Wild, J. R. (1997). Organophosphorus hydrolase is a remarkably stable enzyme that unfolds through a homodimeric intermediate. Biochemistry, 36, 14366-14374.

Guez-Ivanier, V. \& Bedouelle, H. (1996). Disordered C-terminal domain of tyrosyl transfer-RNA synthetase: evidence for a folded state. J. Mol. Biol. 255, 110-120.

Guez-Ivanier, V., Hermann, M., Baldwin, D. \& Bedouelle, H. (1993). Mapping the stability determinants of bacterial tyrosyl transfer-RNA synthetases by an experimental evolutionary approach. J. Mol. Biol. 234, 209-221.

Hardies, S. C. \& Garvin, L. D. (1991). Can molecular evolution provide clues to the folding code? In Conformation and Forces in Protein Folding (Nall, B. T. \& Dill, K. A., eds), vol. 69-76, American Association for the Advancement of Science, Washington.

Heringa, J. \& Argos, P. (1991). Side-chain clusters in protein structures and their role in protein folding. J. Mol. Biol. 220, 151-171.
Heringa, J., Argos, P., Egmond, M. R. \& de Vlieg, J. (1995). Increasing thermal stability of subtilisin from mutations suggested by strongly interacting sidechain clusters. Protein Eng. 8, 21-30.

Kunkel, T. A., Roberts, J. D. \& Zakour, R. A. (1987). Rapid and efficient site-specific mutagenesis without phenotypic selection. Methods Enzymol. 154, 367382.

Malcolm, B. A., Wilson, K. P., Matthews, B. W., Kirsch, J. F. \& Wilson, A. C. (1990). Ancestral lysozymes reconstructed, neutrality tested, and thermostability linked to hydrocarbon packing. Nature, 345, 86-89.

Matthews, B. W. (1993). Structural and genetic analysis of protein stability. Annu. Rev. Biochem. 62, 139-160.

Mildvan, A. S., Weber, D. J. \& Kuliopulos, A. (1992). Quantitative interpretations of double mutations of enzymes. Arch. Biochem. Biophys. 294, 327-340.

Myers, J. K., Pace, C. N. \& Scholtz, J. M. (1995). Denaturant $\mathrm{m}$ values and heat capacity changes: relation to changes in accessible surface areas of protein unfolding. Protein Sci. 4, 2138-2148 and 5, 981.

Neet, K. E. \& Timm, D. E. (1994). Conformational stability of dimeric proteins: quantitative studies by equilibrium denaturation. Protein Sci. 3, 2167-2174.

Ninio, J. (1996). Gene conversion as a focusing mechanism for correlated mutations: a hypothesis. Mol. Gen. Genet. 251, 503-508.

Pace, C. N. (1986). Determination and analysis of urea and guanidine hydrochloride denaturation curves. Methods Enzymol. 131, 266-280.

Pappenberger, G., Schurig, H. \& Jaenicke, R. (1997). Distribution of an ionic network leads to accelerated thermal denaturation of D-glyceraldehyde-3-phosphate dehydrogenase from the hyperthermophilic bacterium Thermotoga maritima. J. Mol. Biol. 274, 676683.

Park, Y. C. \& Bedouelle, H. (1998). Dimeric tyrosyltRNA synthetase from Bacillus stearothermophilus unfolds through a monomeric intermediate. A quantitative analysis under equilibrium conditions. J. Biol. Chem. 273, 18052-18059.

Roder, H. \& Colon, W. (1997). Kinetic role of early intermediates in protein folding. Curr. Opin. Struct. Biol. 7, 15-28.

Shortle, D. (1989). Probing the determinants of protein folding and stability with amino acid substitutions. J. Biol. Chem. 264, 5315-5318.

Sturtevant, J. M. (1994). The thermodynamic effects of protein mutations. Curr. Opin. Struct. Biol. 4, 69-78.

Vidal-Cros, A. \& Bedouelle, H. (1992). Role of residue Glu152 in the discrimination between transfer RNAs by tyrosyl-tRNA synthetase from Bacillus stearothermophilus. J. Mol. Biol. 223, 801-810.

Ward, W. H. \& Fersht, A. R. (1988). Asymmetry of tyrosyl-tRNA synthetase in solution. Biochemistry, 27, 1041-1049.

Waye, M. M., Winter, G., Wilkinson, A. J. \& Fersht, A. R. (1983). Deletion mutagenesis using an 'M13 splint': the N-terminal structural domain of tyrosyl-tRNA synthetase (B. stearothermophilus) catalyses the formation of tyrosyl adenylate. EMBO J. 2, 1827-1829.

Wilkinson, A. J., Fersht, A. R., Blow, D. M. \& Winter, G. (1983). Site-directed mutagenesis as a probe of enzyme structure and catalysis: tyrosyl-tRNA synthetase cysteine-35 to glycine-35 mutation. Biochemistry, 22, 3581-3586.

Wilkinson, A. J., Fersht, A. R., Blow, D. M., Carter, P. \& Winter, G. (1984). A large increase in enzyme-sub- 
strate affinity by protein engineering. Nature, 307, 187-188.

Zhang, H., Skinner, M. M., Sandberg, W. S., Wang, A. H. \& Terwilliger, T. C. (1996). Context depen- dence of mutational effects in a protein: the crystal structures of the V35I, I47V and V35I/I47V gene $\mathrm{V}$ protein core mutants. J. Mol. Biol. 259, 148-159.

Edited by A. R. Fersht

(Received 1 July 1998; received in revised form 14 December 1998; accepted 2 December 1998) 